\title{
A biomechanical comparison of two screw fixation methods in a Letenneur type I Hoffa fracture
}

\author{
Shu-Hsin Yao', Wei-Ren Su, ${ }^{2,3}$, Kai-Lan Hsu, ${ }^{2,4}$, Yueh Chen ${ }^{5}$, Chih-Kai Hong ${ }^{2}$ and Fa-Chuan Kuan ${ }^{2,4^{*}}$ (D)
}

\begin{abstract}
Background: The treatment of Hoffa fractures is challenging, for which the ideal fixation and approach are still controversial. Osteosynthesis with plate or screws fixation in different trajectories have been described in previous literature. The purpose of this study was to compare the biomechanical strength and stability of two types of screw trajectories used to stabilize displaced coronal fractures of the lateral femoral condyle.

Methods: Sixteen synthetic femurs (Sawbones Pacific Research Laboratories, Vashon, WA) were divided into two groups. A vertical osteotomy was performed to mimic a Letenneur type I Hoffa fracture. Group A $(n=8)$ was fixed with screw in anteroposterior direction (A-P) screws. Group B $(n=8)$ was fixed with crossed screws. Both groups were tested with a nondestructive axial compression aligned with the femur axis. After that, 10,000 cyclic loading tests were applied to the specimen with a force ranging between 200 to $600 \mathrm{~N}$, and the interfragmental displacement was recorded, respectively, after 10, 100, 1000 and 10,000 cycles. Finally, a destructive axial compression test was applied until catastrophic failure.
\end{abstract}

Results: There were no statistical between-group differences in regard to the average axial stiffness, interfragmental displacement, and ultimate failure load. The average axial stiffness of the A-P screw was comparable to that of the crossed screw (361 $\pm 113 \mathrm{~N} / \mathrm{mm}$ vs. $379 \pm 65 \mathrm{~N} / \mathrm{mm}, p=0.753)$. All specimens completed the entire cyclic loading test without catastrophic failure, and the interfragmental displacement after loading for 10,000 cycles was $1.36 \pm$ $0.40 \mathrm{~mm}$ for the A-P screw and $1.29 \pm 0.61 \mathrm{~mm}$ for the crossed screw, there were no statistical differences between the groups $(p=0.823)$. The average ultimate failure loads for the A-P and crossed screws were $1214 \pm 127 \mathrm{~N}$ and $1109 \pm 156 \mathrm{~N}$, respectively $(p=0.172)$.

Conclusions: Based on our in vitro study, the crossed screws can provide comparable mechanical performance as traditional A-P screws in Hoffa fracture fixation. Considering the screws trajectories are commonly determined by the choice of surgical approach, the current study provides support from a biomechanical perspective for the application of crossed screws in direct lateral approach.

Keywords: Distal femur fracture, Hoffa fracture, Internal fixation on, Biomechanics

\footnotetext{
*Correspondence: justoversea@hotmail.com

${ }^{2}$ Department of Orthopaedic Surgery, National Cheng Kung University

Hospital, College of Medicine, National Cheng Kung University, Tainan,

Taiwan

${ }^{4}$ Department of Biomedical Engineering, National Cheng Kung University,

Tainan, Taiwan

Full list of author information is available at the end of the article
}

(c) The Author(s). 2020 Open Access This article is licensed under a Creative Commons Attribution 4.0 International License, which permits use, sharing, adaptation, distribution and reproduction in any medium or format, as long as you give appropriate credit to the original author(s) and the source, provide a link to the Creative Commons licence, and indicate if changes were made. The images or other third party material in this article are included in the article's Creative Commons licence, unless indicated otherwise in a credit line to the material. If material is not included in the article's Creative Commons licence and your intended use is not permitted by statutory regulation or exceeds the permitted use, you will need to obtain permission directly from the copyright holder. To view a copy of this licence, visit http://creativecommons.org/licenses/by/4.0/. The Creative Commons Public Domain Dedication waiver (http://creativecommons.org/publicdomain/zero/1.0/) applies to the data made available in this article, unless otherwise stated in a credit line to the data. 


\section{Background}

The Hoffa fracture, which was described by Hoffa first in 1904 [1], represents a fracture pattern that extends coronally in the distal femoral condyle. This type of intraarticular fracture only accounts for $8.7 \%$ 13\% of fractures occurring in the distal femur, and the incidence rate is higher in the lateral as compared to the medial condyle [2-6]. To prevent misdiagnosis, computerized tomography (CT) has been recommended to those sustaining a high-energy distal femoral fracture [7]. As a result of large shearing force, the Hoffa fracture is susceptible to displacement while bearing weight [8]. Nonoperative treatment is not recommended, given that the outcome is unsatisfactory [9]. The aim of surgical treatment is to establish anatomic reduction and rigid fixation [10-13].

The ideal fixation and approach to Hoffa fractures are still subject to debate [14-17]. Plate fixation has been shown to have better stability as compared to isolated screw fixation; however, some unavoidable risks, including extensive dissection and difficulty in plate contouring, have also been reported $[8,18]$. In contrast, screw fixation offers an alternative option with minimal soft tissue dissection. Different screw trajectories, including A-P screws (anteroposterior direction), P-A screws (posteroanterior direction), or crossed screws have been described in previous studies, and all have their proponents [8, 11, 19-21]. The surgical approach also determines the screw trajectory. Given that the Hoffa fracture is diagnosed in association with $38.1 \%$ of supracondylar-intercondylar femoral fractures [4], a direct lateral approach (DLA) or parapatellar approach (PPA) is typically chosen to treat the associated fractures simultaneously [22, 23]. A-P screws and crossed screws can be smoothly utilized through the use of these approaches. Although P-A screws were shown to have better fixation strength than A-P screws in two biomechanical studies $[8,11]$, an additional posterior approach was necessary. Therefore, A-P screws and crossed screws are still widely used in combined Hoffa and distal femoral fractures. To the best of our knowledge, there are no studies that have directly compared these two types of screw trajectories in terms of biomechanics.

The purpose of this study is to compare the biomechanical characteristics of the A-P screws with the crossed screws in a Hoffa fracture. The hypothesis is that in the load-to-failure and cyclic loading test, the crossed screws will provide comparable mechanical properties to those of A-P screws.

\section{Methods}

\section{Specimen preparation}

Sixteen medium, left side, 4th generation synthetic composite femurs (model 3403, Sawbones, Vashon, WA) were utilized in the study. Each specimen was cut into a
$18 \mathrm{~cm}$ distal segment and divided into two groups of 8 each. Then, the anatomic axis of the femurs were wellaligned in a $6 \mathrm{~cm}$ cylinder tube. Afterwards, the specimen was potted in a cylinder tube using anchoring cement (PMMA).

Each specimen was predrilled prior to the osteotomy to facilitate the subsequent anatomic reduction. Furthermore, a specific prefabricated drilling jig was utilized to ensure the consistency of guide pin placement. Initially, two $3.2 \mathrm{~mm}$ threaded pin were advanced under fluoroscopic guidance. After that, the screw length was determined with a direct measuring gauge. Finally, a $4.9 \mathrm{~mm}$ cannulated drill and a $6.5 \mathrm{~mm}$ tap were used to prepare the two screw holes.

In order to mimic the Letenneur type I Hoffa fracture, an oscillating saw was utilized to perform the lateral condylar osteotomy, which was inferiorly extend from the extraarticular condyle-shaft junction to the articular surface. The fracture line was parallel to the axis of posterior femoral cortex. Then, the fracture was reduced anatomically and secured with one of the two screw trajectories described below. All of the surgical procedures were performed by one surgeon under fluoroscopic guidance to ensure accurate implant length and position.

\section{Group A}

Two parallel $6.5-\mathrm{mm}$ partially threaded cannulated screws (Stryker, Kalamazoo, MI) were placed in the A-P fashion. The screws were inserted from the nonarticular portion of the femoral trochlear and were directed posteriorly across to the fracture line perpendicularly. Then, $60 \mathrm{~mm}$ screws were chosen after measurement with a depth gauge. (Fig. 1).

\section{Group B}

Two parallel $6.5-\mathrm{mm}$ partially threaded cannulated screws were placed in the crossed fashion. The screws were inserted from the nonarticular lateral aspect of the condylar fragment, aiming at an inclination of $30^{\circ}$ proximally and $45^{\circ}$ anteriorly. Through this trajectory, screw penetration to the patella-femoral joint could be prevent. Then, $70 \mathrm{~mm}$ screws were chosen after measurement with a depth gauge. (Fig. 2).

\section{Biomechanical protocol}

A materials-testing machine (AG-X; Shimadzu Corp., Tokyo, Japan) was utilized for the biomechanical tests. The specimen potted in a cylinder tube was secured distally into a fabricated adjustable metal clamp (Fig. 3). Afterwards, a flat stainless steel plate connected to a $5000 \mathrm{~N}$ load cell was centered on the Hoffa fracture to apply an axial compression force. Initially, a $100 \mathrm{~N}$ preload was applied to the specimens at a speed of $2 \mathrm{~mm} /$ $\min$. Then, the load was increased to $300 \mathrm{~N}$ at a speed of 

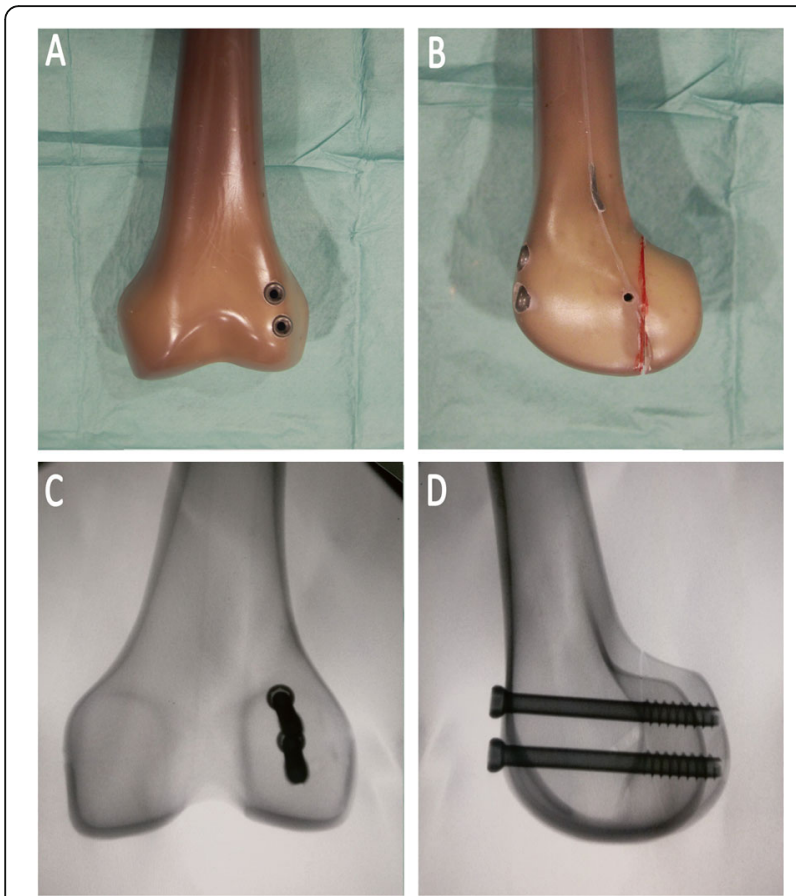

Fig. 1 The specimens were repaired using A-P direction screws. a The dorsal view of the construct; $\mathbf{b}$ the lateral view; c The anteroposterior radiograph view (d) The lateral radiograph view
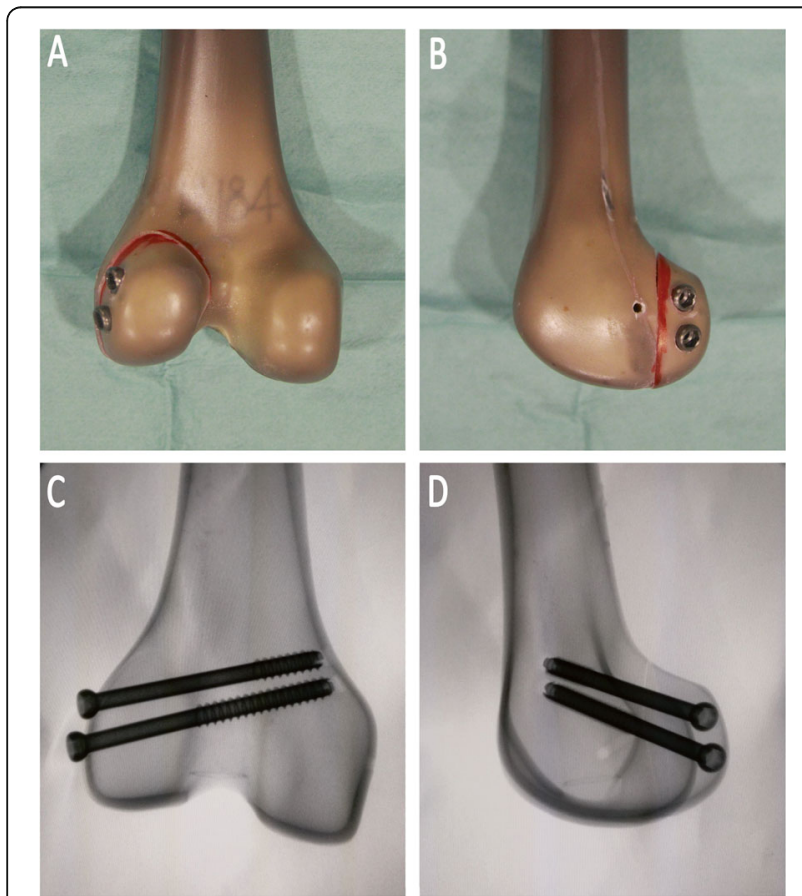

Fig. 2 The specimens were repaired using crossed screws. a The dorsal view of the construct; $\mathbf{b}$ the lateral view; c The posterioanterior radiograph view; d The lateral radiograph view

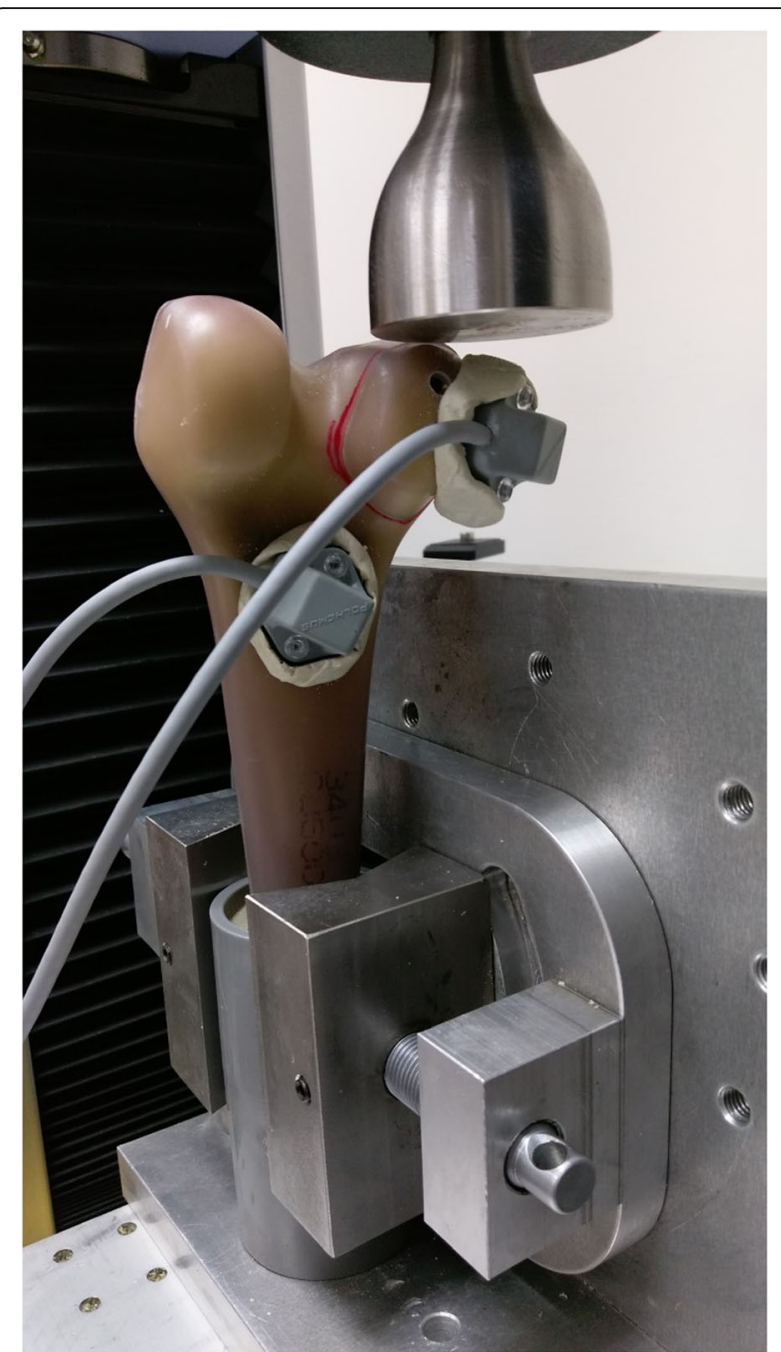

Fig. 3 The biomechanical setup with a composite distal femur mounted on a metal clamp. The displacement sensors were secured with plastic screws and anchoring cement

$10 \mathrm{~mm} / \mathrm{min}$. The slope in the linearly elastic region of the load-displacement curve was recorded and calculated as the axial stiffness. Second, a 10,000 cycle repeated cyclic loading test was applied to the specimen with a force ranging between 200 and $600 \mathrm{~N}$ (valley/peak) at a frequency of $1 \mathrm{~Hz}$, and the interfragmental displacement was recorded, respectively, after 10,100, 1000, and 10, 000 cycles. Finally, destructive axial compression was loaded at a speed of $10 \mathrm{~mm} / \mathrm{min}$ on each specimen until catastrophic failure occurred, which might result from screw dislodging, fragment cracking or cortex disruption. The loading protocol mainly followed a previous study [11]. The maximum load detected by the testing machine during the load-to-failure test was defined as failure load. In addition, all failure modes were recorded for the purpose of the analysis. There were two sensors firmly fixed by cement onto the fragment and intact 
distal femur respectively. All the interfragmental displacement during the static and cyclic loading was measured with a magnetic tracking system (Polhemus, Colchester, VT, USA), by which the real-time data was detected at a resolution of $0.03 \mathrm{~mm}$.

\section{Statistical analysis}

Following the pilot study, in which three specimens in each group was conducted, an priori power analysis was performed using G*Power [24]. The effect sizes were calculated from the preliminary results and were 2.17 for the failure load and 1.18 for the stiffness. To provide a study power of 0.9 and $\alpha$ value of 0.05 , the projected sample size needed with this effect size is approximately 8 for each group. The final data from the two groups was conducted using SPSS software (SPSS Version 17; SPSS Inc., Chicago, IL, USA). To determine the differences in axial stiffness and destructive failure load between the two types of screw trajectories, considering the small sample size, the Mann-Whitney $U$ test was performed for the distribution-free equivalent statistic on the data analysis. As regards the cyclic displacement, considering that repeated measurements of cyclic displacement were made four times on each specimen, a 4step General Linear Model Repeated Measures Test was performed. A confidence interval of $95 \%$ was used for all the parameters to determine significance.

\section{Results}

The raw biomechanical data for axial stiffness, the interfragmental displacement at each specific cycle, and the ultimate failure load are listed in Table 1.

\section{Axial stiffness}

The slope in the linearly elastic region was referred as axial stiffness, which was $361 \pm 113 \mathrm{~N} / \mathrm{mm}$ in the group $A$ and $379 \pm 65 \mathrm{~N} / \mathrm{mm}$ in the group B. There were no significant differences between the groups $(p=0.753)$.

\section{Interfragmental displacement}

The interfragmental fracture displacement after each specific cyclic loading was collected, where it was found that the value increased steadily during the cyclic test. In the group $\mathrm{A}$, the interfragmentary fracture displacement after $10,100,1000$, and 10,000 cycles was $0.69 \pm 0.19$ $\mathrm{mm}, 0.88 \pm 0.23 \mathrm{~mm}, 1.12 \pm 0.25 \mathrm{~mm}, 1.36 \pm 0.40 \mathrm{~mm}$, respectively. In the group $\mathrm{B}$, the interfragmentary fracture displacement after 10,100,1000, and 10,000 cycles was $0.90 \pm 0.48 \mathrm{~mm}, 1.04 \pm 0.55 \mathrm{~mm}, 1.14 \pm 0.62 \mathrm{~mm}, 1.29 \pm$ $0.61 \mathrm{~mm}$, respectively. There were no statistically significant differences between the two fixation constructs after each cycle.

\section{Load to failure}

All of the specimens completed the entire cyclic loading test. Afterwards, the specimens were loaded until catastrophic failure. Based on the load-displacement curve, all the specimens exhibited changes in the curve slope prior to catastrophic failure, which indicated loss of fixation or plastic deformation of the construct. Followed with the plastic deformation, the maximum load detected by the testing machine was defined as the ultimate failure load, which was $1214 \pm 127 \mathrm{~N}$ in the group $\mathrm{A}$ and $1109 \pm 156 \mathrm{~N}$ in the group B. There were no significantly differences between the groups $(p=0.172)$. The most common pattern of failure was screw cut through the cancellous bone progressively, after that, the cortex bone was ruptured while maximum failure load was detected. (Fig. 4).

\section{Discussion}

In this time zero study, the biomechanical properties of two different types of screw trajectories for the treatment of a lateral Hoffa fracture were compared. On the basis of the results, the crossed screw construct exhibited comparable biomechanical properties to those of the A-P screw construct. Therefore, the principle findings of the current study suggest that both screw trajectories will provide sufficient stability in terms of fracture fixation.

Several fixation methods, including screws and plates, have been utilized in the Hoffa fracture treatment. Sun et al. conducted a biomechanical analysis of four fixation constructs, and they concluded that a combination of plate and screw fixation could provide greater biomechanical stability than a screw fixation system alone [8]. In spite of its mechanical superiority, the popularity of plate fixation is still low given the requirement for extensive dissection and blood supply disturbance. Meanwhile, the ideal size and number of screws were discussed in a

Table 1 Average axial stiffness, post cyclic load displacement, and ultimate failure load in the different groups

\begin{tabular}{lllllll}
\hline Groups & Stiffness $(\mathrm{N} / \mathrm{mm})$ & \multicolumn{2}{l}{ Displacement $(\mathrm{mm})$} & \multicolumn{3}{l}{ Failure load (N) } \\
\cline { 3 - 6 } & & 10 cycles & 100 cycles & 1000 cycles & 10,000 cycles & $1.36(0.40)$ \\
\hline A-P & $361(113)$ & $0.69(0.19)$ & $0.88(0.23)$ & $1.12(0.25)$ & $1.36(127)$ \\
Crossed & $379(65)$ & $0.90(0.48)$ & $1.04(0.55)$ & $1.14(0.62)$ & $1.29(0.61)$ & $1109(156)$ \\
$p$-value & 0.753 & 0.265 & 0.454 & 0.938 & 0.823 & 0.172 \\
\hline
\end{tabular}

Average values are given along with one standard deviation in parentheses 


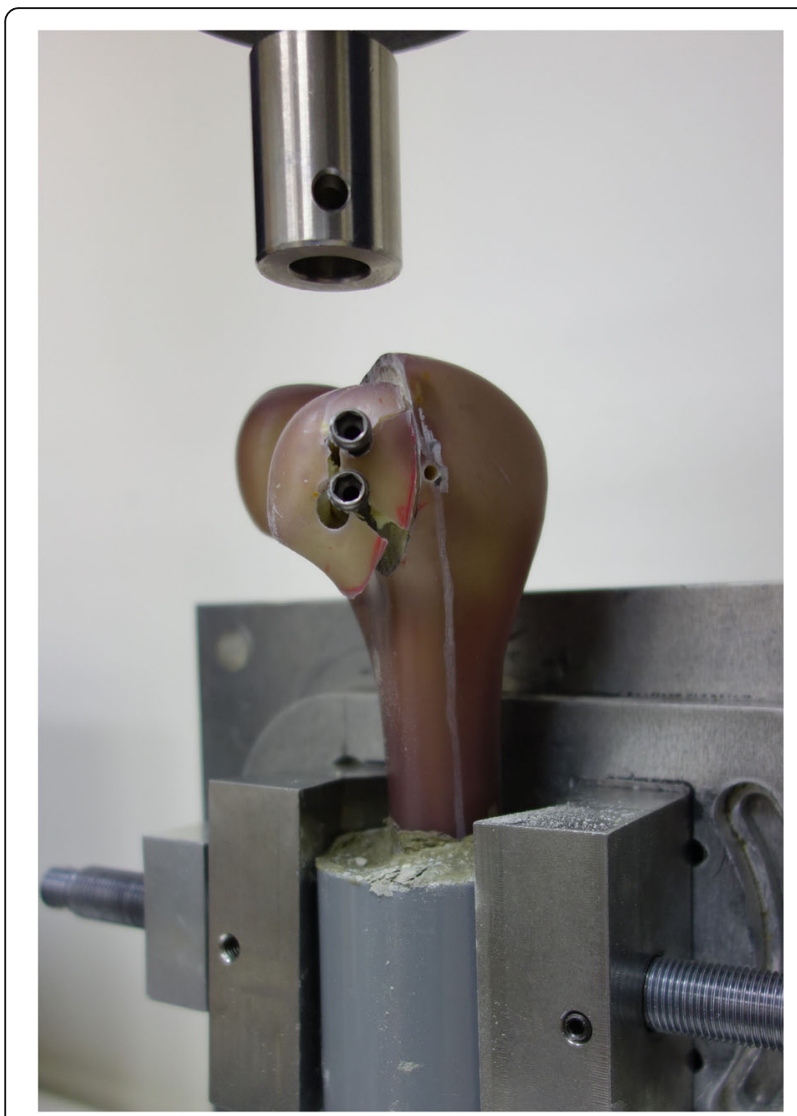

Fig. 4 The Hoffa fragment was displaced distally from its original position. Most of the constructs failed as the screw dislodging accompanied with cortex disruption

previous study [12]. A cadaveric study compared the biomechanical characteristics for three sizes of screws in a Hoffa fracture fixation, where the ultimate failure was significantly higher for $6.5-\mathrm{mm}$ cancellous screws as compared to $3.5-\mathrm{mm}$ cortical screws [21]. Furthermore, a biomechanical study showed that single $6.5 \mathrm{~mm}$ screws provide better fixation than either single or double 3.5 $\mathrm{mm}$ screws, yet a two screw construct is suggested to achieve two points of fixation in order to prevent fragment rotation [12]. Therefore, we utilized two $6.5 \mathrm{~mm}$ partially threaded screws in different trajectories in the current study.

The ideal screw trajectory has been discussed widely in previous studies for A-P screws, P-A screws, and crossed screws [19-21]. A biomechanical study conducted by Jarit et al. demonstrated that screws positioned in the P-A fashion exhibit significantly higher stability than A-P screws [11]. However, it is difficult to insert a P-A screw perpendicularly to a Hoffa fracture using a direct lateral approach. Therefore, an additional posterior approach is necessary [22], which inevitably increases the risk of common peroneal nerve injury or a compromise in blood supply [23]. In addition, fixation using a P-A screw necessitates advancing screws through the posterior condylar cartilage area, which will damage the weight-bearing articular surface [23]. Alternatively, $\mathrm{Xu}$ et al. treated eleven Hoffa fracture patients using crossed screws, and a comparison with traditional screws showed no significant differences in the function outcomes after 2 years of follow-up, which might indicate that crossed screws are as effective as traditional screws $[19,20]$.

The surgical approach depends on the pattern of the fracture or surgeon preference. When a Hoffa fracture occurs in association with a distal femoral fracture, both surgical approaches, including the direct lateral or lateral parapatellar approach, are commonly used. For fixation of a Hoffa fracture, an A-P screw is easier to apply via the parapatellar approach. However, an anatomic study showed that when the Hoffa fragment is less than $10.1 \%$ of the AP diameter of the lateral condyle, the parapatellar approach does not allow direct visualization of the fracture [23], which makes reduction difficult and may result in unsatisfactory results. In contrast, the direct lateral approach may provide better access to the Hoffa fracture. Shi et al. reported excellent results in 12 isolated lateral Hoffa fractures treated through the direct lateral approach [18]. In addition, a crossed screw could be inserted from the nonarticular area of the fractured condylar fragment in this approach. On the basis of our results, it was determined that a crossed screw may offer similar fixation strength as compared to a traditional A-P screw.

There are some limitations to this study. First, the fracture model was set in a synthetic bone rather than in a cadaveric femur, which might be better to simulate an in vivo situation. However, the uniform geometry of synthetic bone could minimize interspecimen variations in the physical properties of the bone. Also, the synthetic composite femur utilized in the current study was designed in accordance with the mechanical properties of a healthy male $<60$ years of age [25]. Given that the current study was mainly aimed at high-energy trauma occurring in a younger population, synthetic bone could prevent the complications associated with inherent osteoporosis that might be a characteristic of cadaveric specimens. Second, not all of the force components were included in the biomechanical test. For example, bending and torsion tests were not conducted in the current study. Finally, P-A screws or plates were not included in the current study. As described previously, we focused on the crossed screw trajectory because it could be inserted using a direct lateral approach, and given that a comparison of A-P and PA screws was made in a previous study, too many groups in the study might have led to confusing results.

\section{Conclusion}

Based on our in vitro study, the crossed screws can provide comparable mechanical performance as traditional 
A-P screws in Hoffa fracture fixation. Considering the screws trajectories are commonly determined by the choice of surgical approach, the current study provides support from a biomechanical perspective for the application of crossed screws in direct lateral approach.

\section{Abbreviations}

A-P screw: Anteroposterior direction screw; P-A screw: Posteroanterior direction screw; DLA: Direct lateral approach; PPA: Parapatellar approach

\section{Acknowledgements}

We thank Skeleton Materials and Bio-compatibility Core Lab, Research Center of Clinical Medicine, National Cheng Kung University Hospital, College of Medicine, National Cheng Kung University for assistance with this project.

\section{Authors' contributions}

SHY: study design, data acquisition, analyses and interpretation of data, draft of manuscripts, tables and figures. WRS; study design, data acquisition, analyses and interpretation of data. KLH; analyses and interpretation of data, manuscript with tables and figures. YC; study design, data acquisition, analyses and interpretation of data, manuscript with tables and figures. $\mathrm{CKH}$; draft of manuscripts, tables and figures. FCK; study design, data acquisition, analyses and interpretation of data, manuscript with tables and figures. All authors critically reviewed and approved the final revised manuscript.

\section{Funding}

This project was funded by a grant from National Cheng Kung University Hospital (grants: NCKUH-10804007).

\section{Availability of data and materials}

All relevant data supporting the conclusions are included within the article and tables. The datasets used and/or analysed during the current study available from the corresponding author on reasonable request.

\section{Ethics approval and consent to participate}

Not applicable.

\section{Consent for publication}

Not applicable.

\section{Competing interests}

The authors declare that they have no competing interests.

\section{Author details}

'Department of Orthopaedic Surgery, Ditmanson Medical Foundation Chia Yi Christian Hospital, Chiayi, Taiwan. ${ }^{2}$ Department of Orthopaedic Surgery, National Cheng Kung University Hospital, College of Medicine, National Cheng Kung University, Tainan, Taiwan. ${ }^{3}$ Skeleton Materials and Bio-compatibility Core Lab, Research Center of Clinical Medicine, National Cheng Kung University Hospital, College of Medicine, National Cheng Kung University, Tainan, Taiwan. ${ }^{4}$ Department of Biomedical Engineering, National Cheng Kung University, Tainan, Taiwan. ${ }^{5}$ Department of Orthopaedic Surgery, Sin Lau Hospital, Tainan, Taiwan.

Received: 7 January 2020 Accepted: 20 July 2020

Published online: 28 July 2020

References

1. Hoffa A. Lehrbuch der Frakturen und Luxationen. 4th ed. Stuttgart: Ferdinand Enke; 1904

2. Zhou Y, Pan Y, Wang Q, Hou Z, Chen W. Hoffa fracture of the femoral condyle: injury mechanism, classification, diagnosis, and treatment. Medicine. 2019;98:8.

3. Mandredini M, Gildone A, Ferrante R, Bernasconi S, Massari L. Unicondylar femoral fractures: therapeutic strategy and long-term results. A review of 23 patients. Acta Orthop Belg. 2001;67(2):132-8.

4. Nork SE, Segina DN, Aflatoon K, Barei DP, Henley MB, Holt S, et al. The association between supracondylar-intercondylar distal femoral fractures and coronal plane fractures. JBJS. 2005;87(3):564-9.
5. Lewis S, Pozo J, Muirhead-Allwood W. Coronal fractures of the lateral femoral condyle. J Bone Joint Surg Br Vol. 1989:71(1):118-20.

6. Jain A, Aggarwal P, Pankaj A. Concomitant ipsilateral proximal tibia and femoral Hoffa fractures. Acta Orthop Traumatol Turc. 2014;48(4):383-7.

7. Arastu M, Kokke M, Duffy P, Korley R, Buckley R. Coronal plane partial articular fractures of the distal femoral condyle: current concepts in management. Bone Joint J. 2013;95(9):1165-71.

8. Sun H, He Q-F, Huang Y-G, Pan J-F, Luo C-F, Chai Y-M. Plate fixation for Letenneur type I Hoffa fracture: a biomechanical study. Injury. 2017:48(7): 1492-8.

9. Chang JJHT, Fan JCH, Lam HY, Cheung KY, Chu WWS, Fung KY. Treatment of an osteoporotic Hoffa fracture. Knee Surg Sports Traumatol Arthrosc. 2010; 18(6):784-6

10. Xie $X$, Zhan $Y$, Dong M, He Q, Lucas JF, Zhang Y, et al. Two and threedimensional CT mapping of Hoffa fractures. JBJS. 2017:99(21):1866-74.

11. Jarit GJ, Kummer FJ, Gibber MJ, Egol KA. A mechanical evaluation of two fixation methods using cancellous screws for coronal fractures of the lateral condyle of the distal femur (OTA type 33B). J Orthop Trauma. 2006;20(4): 273-6.

12. Hak DJ, Nguyen J, Curtiss S, Hazelwood S. Coronal fractures of the distal femoral condyle: a biomechanical evaluation of four internal fixation constructs. Injury. 2005:36(9):1103-6.

13. Ostermann P, Neumann K, Ekkernkamp A, Muhr G. Long term results of unicondylar fractures of the femur. J Orthop Trauma. 1994:8(2):142-6.

14. Heuschen U, Göhring U, Meeder P. Bilateral Hoffa fracture--a rarity; 1994.

15. Holmes SM, Bomback D, Baumgaertner MR. Coronal fractures of the femoral condyle: a brief report of five cases. J Orthop Trauma. 2004;18(5):316-9.

16. Calmet J, Mellado J, Forcada IG, Giné J. Open bicondylar Hoffa fracture associated with extensor mechanism injury. J Orthop Trauma. 2004;18(5): 323-5.

17. Butler MS, Brumback R, Ellison T, Poka A, Bathon G, Burgess A. Interlocking intramedullary nailing for ipsilateral fractures of the femoral shaft and distal part of the femur. J Bone Joint Surg Am. 1991:73(10):1492-502.

18. Shi J, Tao J, Zhou Z, Gao M. Surgical treatment of lateral Hoffa fracture with a locking plate through the lateral approach. Eur J Orthop Surg Traumatol. 2014;24(4):587-92

19. $\mathrm{Xu}$ Y, Li $\mathrm{H}$, Yang $\mathrm{H}-\mathrm{H}$. A new fixation method for Hoffa fracture. Eur J Trauma Emerg Surg. 2013;39(1):87-91

20. Xu Y, Li H. Yang H-h, Pan Z-j: a comparison of the clinical effect of two fixation methods on Hoffa fractures. Springerplus. 2016;5(1):1164.

21. Becker P. Comparative analysis for the fixation of coronal distal intraarticular femur fractures. In: The 67th annual meeting of the American Academy of Orthopaedic surgeons, march 15-19, 2000; 2000

22. Orapiriyakul W, Apivatthakakul T, Buranaphatthana T. How to determine the surgical approach in Hoffa fractures? Injury. 2018;49(12):2302-11.

23. Orapiriyakul W, Apivatthakakul T, Phornphutkul C. Relationships between Hoffa fragment size and surgical approach selection: a cadaveric study. Arch Orthop Trauma Surg. 2018;138(12):1679-89.

24. Faul F, Erdfelder E, Lang AG, Buchner A. G*power 3: a flexible statistical power analysis program for the social, behavioral, and biomedical sciences. Behav Res Methods. 2007;39(2):175-91.

25. Gardner MP, Chong AC, Pollock AG, Wooley PH. Mechanical evaluation of large-size fourth-generation composite femur and tibia models. Ann Biomed Eng. 2010;38(3):613-20.

\section{Publisher's Note}

Springer Nature remains neutral with regard to jurisdictional claims in published maps and institutional affiliations. 